\title{
Superficial Musculoaponeurotic System Elevation and Fat Graft Reconstruction After Superficial Parotidectomy
}

\author{
Joseph M. Curry, MD; Kyle W. Fisher, MD; Ryan N. Heffelfinger, MD; Marc R. Rosen, MD;
} William M. Keane, MD; Edmund A. Pribitkin, MD

Objective/Hypothesis: Elevation of the superficial musculoaponeurotic system (SMAS) with or without fat graft interposition during superficial parotidectomy prevents a concave facial deformity and Frey's syndrome.

Study Design: Retrospective, case-control study.

Methods: Charts for 248 patients who underwent superficial parotidectomy were reviewed for pathologic, radiographic, clinical, and operative data. Sixteen patients who underwent SMAS elevation and 34 patients who underwent SMAS elevation with fat graft interposition were included in two study groups. Nonreconstructed controls were randomly selected from a pool of patients who had unilateral, superficial parotidectomy and were matched based on pathologic specimen volume. Patients were surveyed for their postoperative symptoms.

Results: Patients undergoing SMAS elevation alone $(\mathrm{n}=16)$ compared with controls $(\mathrm{n}=19)$ had greater facial symmetry ( $12 \%$ vs. $32 \%, P=.147)$ and a lower incidence of symptomatic Frey's syndrome (6.3\% vs. $18.6 \%, P=.382$ ). Patients undergoing SMAS elevation and fat graft interposition $(\mathrm{n}=34)$ compared with controls $(\mathrm{n}=38)$ had less facial asymmetry $(9 \%$ vs. $39 \%, P=$ .002 ) and a lower incidence of symptomatic Frey's syndrome ( $6 \%$ vs. $28 \%, P=.04$ ). Complications among the study and control groups were comparable.

Conclusions: Simultaneous reconstruction of a superficial parotidectomy defect using SMAS elevation with or without fat grafting may improve postoperative facial symmetry and decrease the incidence of symptomatic Frey's syndrome without increasing complications.

From the Department of Otolaryngology Head and Neck Surgery, Thomas Jefferson University, Philadelphia, Pennsylvania, U.S.A. 10, 2007.

Editor's Note: This Manuscript was accepted for publication August

Presented at Triological Society 2007 Combined Section Meeting, Marco Island, Florida, U.S.A., February 14-18, 2007.

Disclosure: No financial support was received for this project.

Send correspondence to Dr. Joseph M. Curry, Department of Otolaryngology Head and Neck Surgery, Thomas Jefferson University, 925 Chestnut Street, 6th Floor, Philadelphia, PA 19107. E-mail: joseph.curry@jefferson.edu

DOI: 10.1097/MLG.0b013e3181581f94
Key Words: Superficial musculoaponeurotic system, parotidectomy fat graft, reconstruction.

Laryngoscope, 118:210-215, 2008

\section{INTRODUCTION}

Adequate tumor resection and preservation of facial nerve function are the primary goals of superficial parotidectomy, yet this surgery may lead to undesirable effects, including a concave facial defect, Frey's syndrome, and prominent scar. Commonly, a depression over the mandibular angle or pre-auricular region is noticeable, and several methods have been described to prevent such defects, including superficial musculoaponeurotic system (SMAS) plication, sternocleidomastoid (SCM) muscle flap repair, and free dermal-fat grafting. ${ }^{1-5}$ SMAS plication can be effective for small defects. Fat grafts have long been used to improve facial defects, but their primary limitation has been resorption. ${ }^{4-6}$

Furthermore, reconstructive techniques may also decrease the symptoms of Frey's syndrome. The reported incidence after parotidectomy varies, but clinical symptoms may be evident in $10 \%$ to $30 \%$ of patients, whereas starch-iodine tests may be positive in up to $90 \% .^{2,7}$ Symptoms can be prevented by interposing a tissue or graft layer between regenerating parasympathetic neurons and sweat glands, thereby blocking aberrant reinnervation. ${ }^{8}$ To prevent a prominent scar, the use of a rhytidectomy incision has been described, which improves cosmesis and provides adequate exposure. ${ }^{9}$ This study reviews the outcomes of facial symmetry and symptomatic Frey's syndrome in patients having simultaneous SMAS elevation with or without fat grafting during superficial parotidectomy.

\section{MATERIALS AND METHODS}

This study was approved by the institutional review board of our institution. A retrospective chart review of 248 patients who underwent superficial parotidectomy between October 1998 and June 2006 was performed. Patients included underwent a single, unilateral, superficial parotidectomy for benign disease without history of contralateral parotidectomy. Subsequently, a telephone survey was performed to score cosmetic satisfaction 
and postoperative symptoms on a 4-point scale (no symptoms, mild, moderate, severe symptoms).

Patients undergoing superficial parotidectomy with SMAS elevation comprised the first study group; patients reconstructed with SMAS elevation and fat grafting comprised the second study group. Control groups were established by randomly selecting volume-matched individuals from patients who had superficial parotidectomy without reconstruction. Volume matching was performed using the calculated surgical specimen volume (all parotid tissue removed at surgery) based on surgical pathology report measurements. Results for facial asymmetry and symptoms of Frey's syndrome were statistically analyzed using the $\chi^{2}$, Wilcoxon, and proportional odds ratio tests.

\section{Surgical Procedure}

In patients undergoing reconstruction, a modified rhytidectomy incision is used (Fig. 1). The preauricular incision follows the line of the incisura, curves around the lobule and follows the postauricular crease, and then descends $1 \mathrm{~cm}$ posterior to the
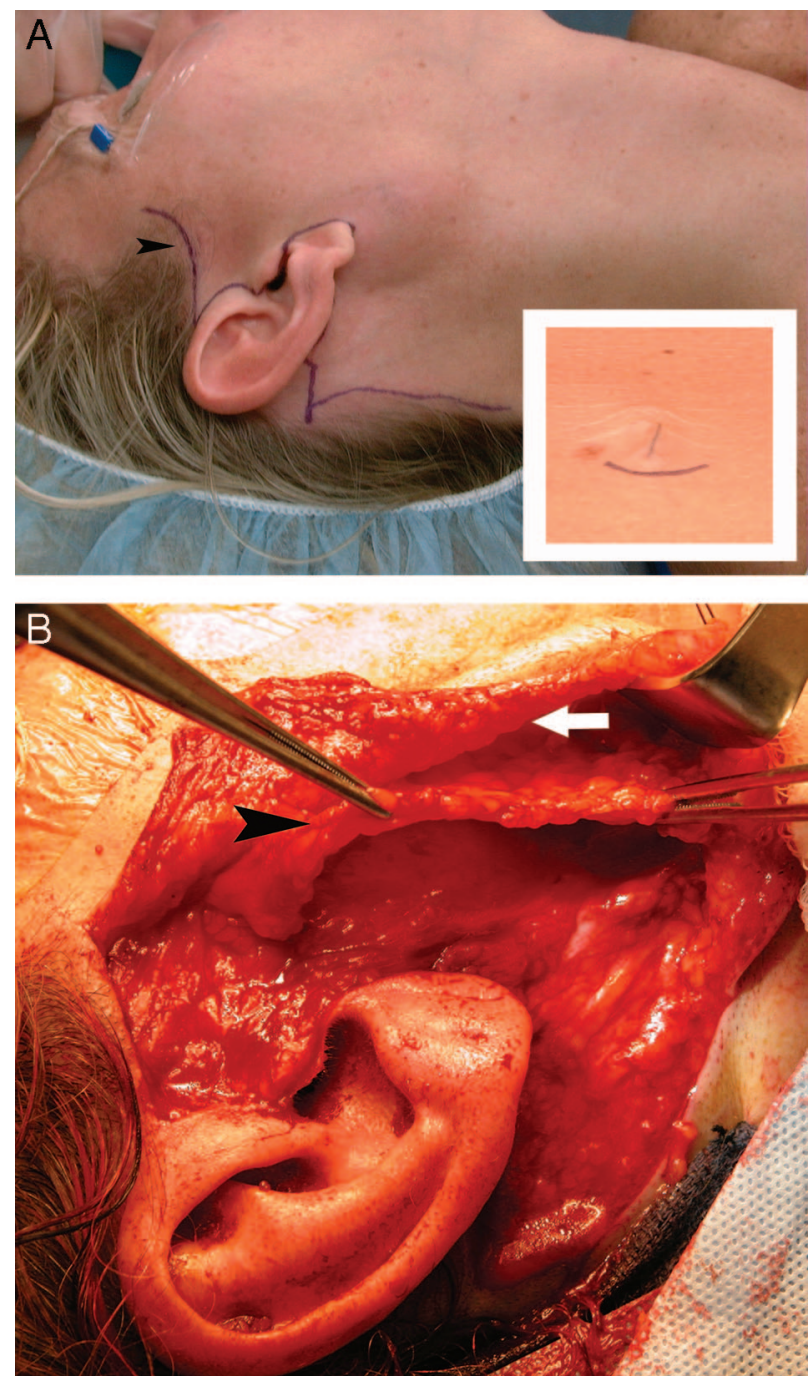

Fig. 1. (A) Rhytidectomy incision used for parotidectomy. A horizontal anterior limb can be added at the sideburn to increase exposure (black arrowhead). The inset shows the infraumbilical incision for fat graft. Elevation of the skin flap in the subcutaneous plane (white arrow) and superficial musculoaponeurotic system layer (black arrowhead) (B). hairline. The incision can be extended anteriorly $1 \mathrm{~cm}$ superior to the temporal hairline or inferiorly into a transverse neck crease if necessary for exposure. The skin flap is raised in the subcutaneous plane superficial to the SMAS to obtain adequate exposure for tumor removal. The SMAS and contiguous platysma muscle are elevated as a separate layer while care is taken not to violate the underlying parotid fascia. If the tumor involves the SMAS layer, the affected portion of this layer is excised with the specimen, and the defect is repaired at closure.

Facial nerve dissection and tumor removal is performed, followed by intraoperative frozen section to confirm benign disease. Afterward, the need for fat grafting is determined based on the defect volume. A single fat graft, designed to overcorrect the defect volume by $15 \%$ to $20 \%$, is harvested from the infraumbilical region through a $2.0 \mathrm{~cm}$ incision (Fig. 1). The graft is rinsed in normal saline and sutured into place, with care taken to minimize manipulation. The SMAS layer is returned to its original position over the fat graft and secured with absorbable suture. A suction drain is placed, and the incision is closed.

\section{RESULTS}

In the SMAS elevation group, 16 of 18 patients were contacted (Fig. 2). The mean volume of their pathologic specimen was $7.9 \mathrm{~cm}^{3}$, and their average follow-up was 22.7 months (Table I). Two (12\%) patients complained of facial asymmetry, which they both scored as mild (Table II). Neither sought correction. For volume-matched controls, 19 patients were contacted. The mean volume of

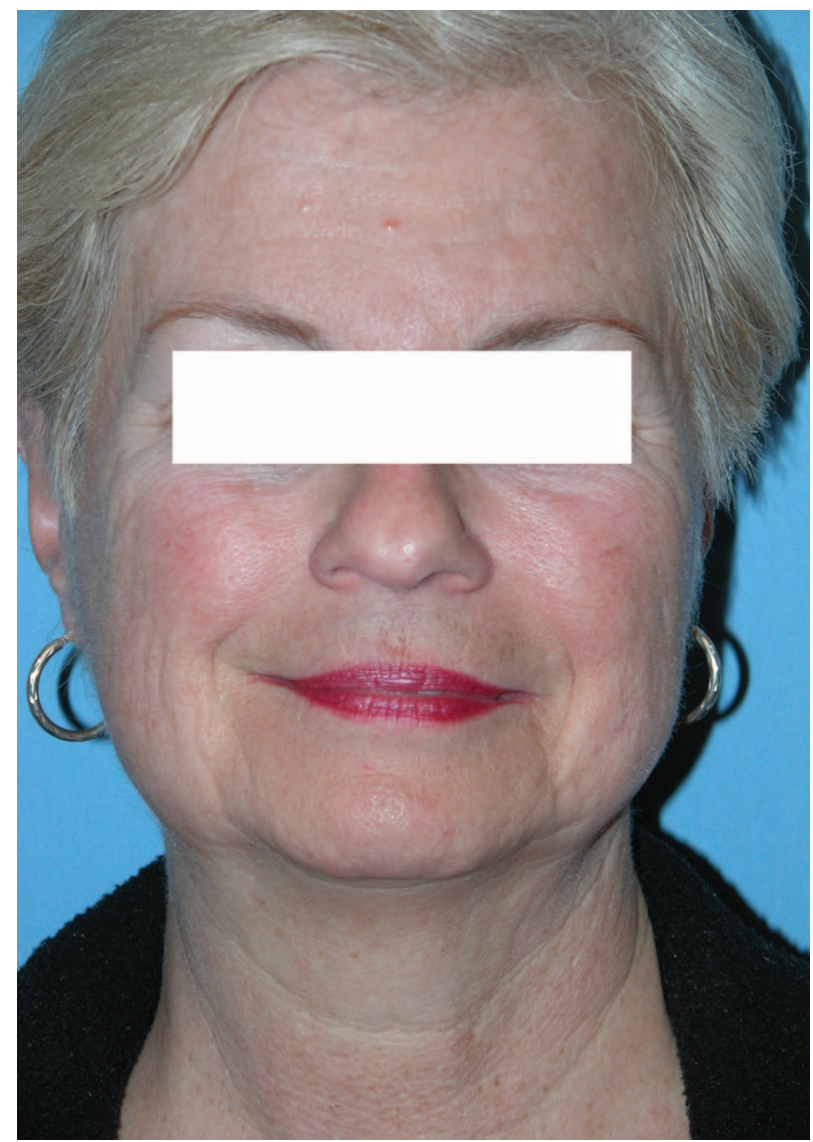

Fig. 2. Frontal view of patient status post right parotidectomy with superficial musculoaponeurotic system elevation for reconstruction approximately 2 months after surgery. 
TABLE I.

Patient Data, Pathology, and Radiographic Findings.

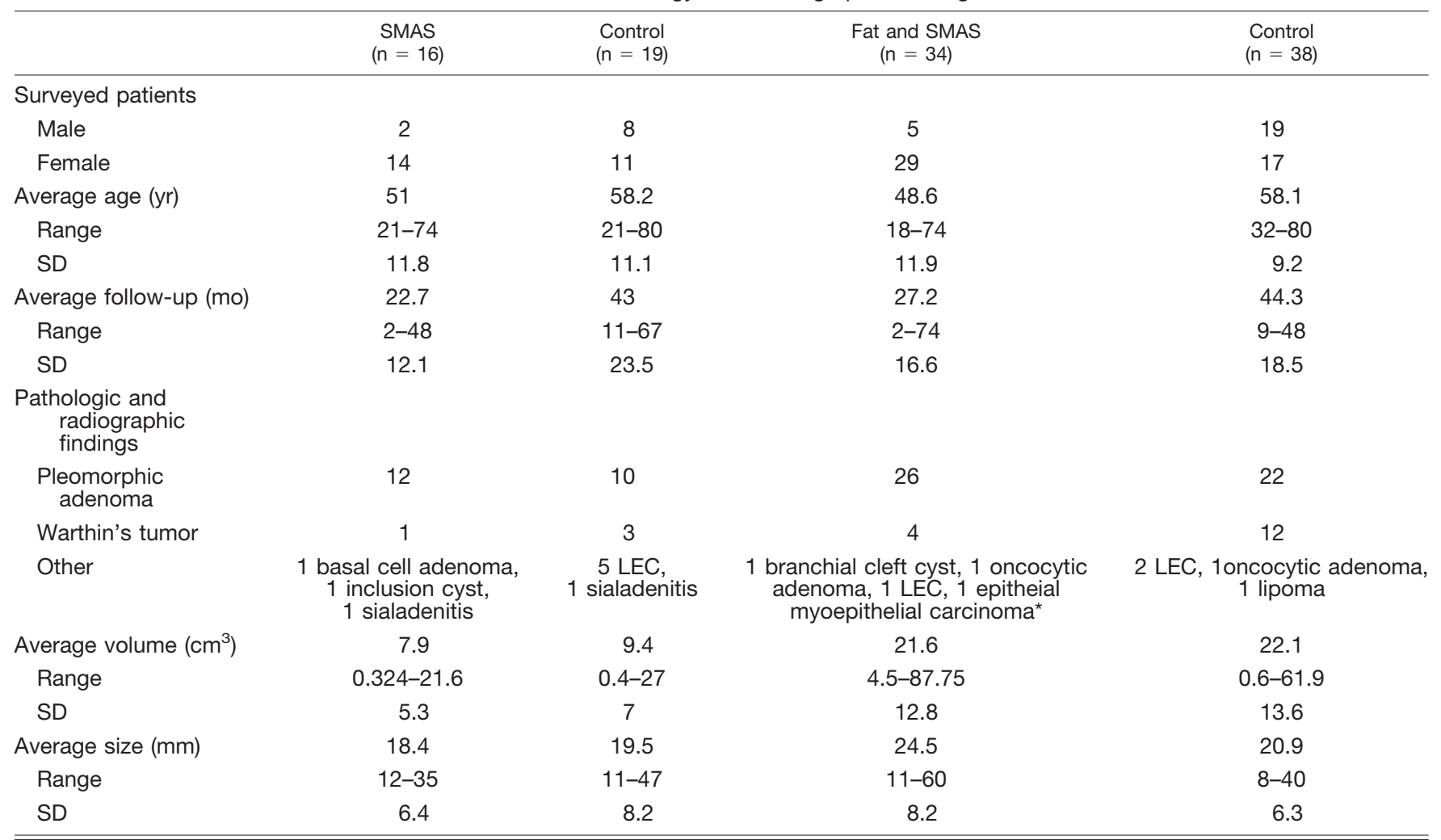

SMAS = superficial musculoaponeurotic system; LEC = lymphepithelial cyst.

*Preoperative FNA and intraoperative frozen section consistent with benign disease.

their pathologic specimens was $9.4 \mathrm{~cm}^{3}$, and their average follow-up was 43 months. In this group, six (32\%) patients complained of asymmetry: four noted slight, one moderate, and one severe asymmetry. One patient sought treatment. Overall, patients undergoing SMAS elevation reported greater facial symmetry than their counterpart control group, but this difference was not statistically significant $\left(P=.147, \chi^{2}\right.$ test). The odds ratio for facial symmetry between the two groups was $3.01(P=.244)$.

In this study group, one (6.3\%) patient experienced moderate symptoms of Frey's syndrome versus three $(18.6 \%)$ in the control group (Table II). Two of the patients had mild symptoms, and one had severe symptoms. The patient with severe symptoms expressed interest in treatment. Patients in the study group reported a lower incidence of Frey's syndrome than did those in the control group, although this difference was not statistically significant $\left(P=.382, \chi^{2}\right.$ test). The calculated odds ratio was $2.54(P=.608)$.

Complications within these two groups were comparable. In the study group, one patient developed transient facial weakness, and no patients developed infections or collections. In the control group, no patients had transient facial weakness, and one developed a hematoma. No patients in either group developed permanent facial weakness. One patient in the study group and four control patients complained of prominent scar. Eight patients in each group complained of auricular numbness.
Of the 37 patients who had SMAS elevation and fat graft, 34 were contacted (Fig. 3). The average pathologic specimen volume was $21.6 \mathrm{~cm}^{3}$, and their average follow-up was 27.2 months (Table I). Thirty-one of 34 perceived their facial contour as symmetric, and $3(9 \%)$ patients thought that their faces were asymmetric (Table II). Two patients complained of mild asymmetry, and one complained of moderate asymmetry. One patient sought treatment. The patient with moderate asymmetry was surveyed at 29 months postoperatively, and the two with mild asymmetry were surveyed at 10 and 45 months postoperatively. Thirty-eight patients were contacted for the control group; their average pathologic specimen volume was $22.1 \mathrm{~cm}^{3}$, and their average follow-up was 44.3 months. In this group, $15(39 \%)$ patients complained of facial asymmetry. Ten complained of mild asymmetry, and four noted moderate asymmetry. Two patients expressed interest in treatment. In summary, patients undergoing SMAS elevation with fat graft reported significantly greater facial symmetry than volume-matched controls $\left(P=.002, \chi^{2}\right.$ test $)$. The odds ratio for facial symmetry between these two groups was $6.35(P=.003)$.

In this study group, two (6\%) patients complained of mild symptoms of Frey's syndrome. None of these patients sought treatment. In the control group, eight (28\%) patients developed symptoms of Frey's syndrome. Three had mild symptoms, two had moderate, and three had severe symptoms. Two of these patients sought treatment. Over- 
TABLE II

Survey Results.

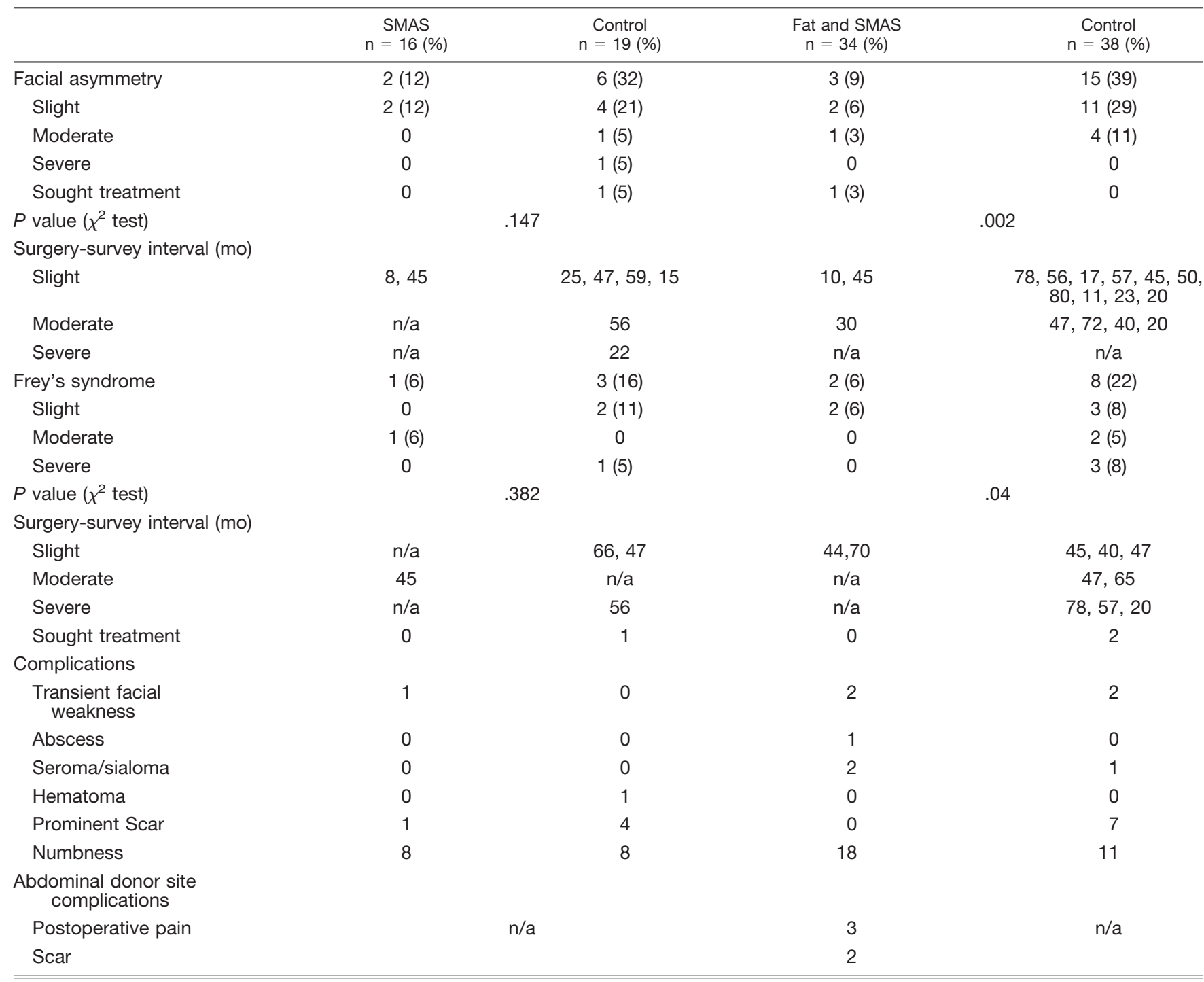

SMAS = superficial musculoaponeurotic system.

all, reconstructed patients noted a statistically significant decrease in symptoms of Frey's syndrome compared with those in the control group $\left(P=.04, \chi^{2}\right.$ test). The odds ratio for this study group was $4.123(P=.087)$.

Complications among these two groups were comparable (Table II). In the study group, two patients developed slight, transient facial weakness compared with two in the control group (Table II). Two patients subsequently developed a sialoma that resolved after several aspirations. In the control group, one patient developed sialoma. The most common complaint was auricular numbness, noted by 18 patients versus 11 patients in the control group. Three patients complained of significant pain at the abdominal site, which resolved after the immediate postoperative period. Two patients complained of noticeable abdominal scar.

One patient in the fat grafted group had fine-needle aspiration (FNA) and frozen section suggestive of benign disease; however, final pathology revealed epithelialmyoepithelial carcinoma. This patient has been followed with clinical examination and magnetic resonance imaging (MRI) and has not developed recurrence.

\section{DISCUSSION}

This study demonstrated a statistically significant decrease in asymmetry and symptoms of Frey's syndrome after SMAS elevation with fat grafting. There was a decrease in asymmetry and Frey's syndrome in the SMAS elevation group, but this was not statistically significant.

Multiple techniques are available for reconstruction of parotidectomy defects. The SMAS can be rotated from the cervical region to fill a defect, as described by Rappaport and Allison. ${ }^{1}$ It also can be plicated to fill a preauricular defect, as described by Casler and Conley. ${ }^{2}$ It has not been our practice to plicate or rotate the SMAS but rather redrape it over smaller defects. In this study, the patients 


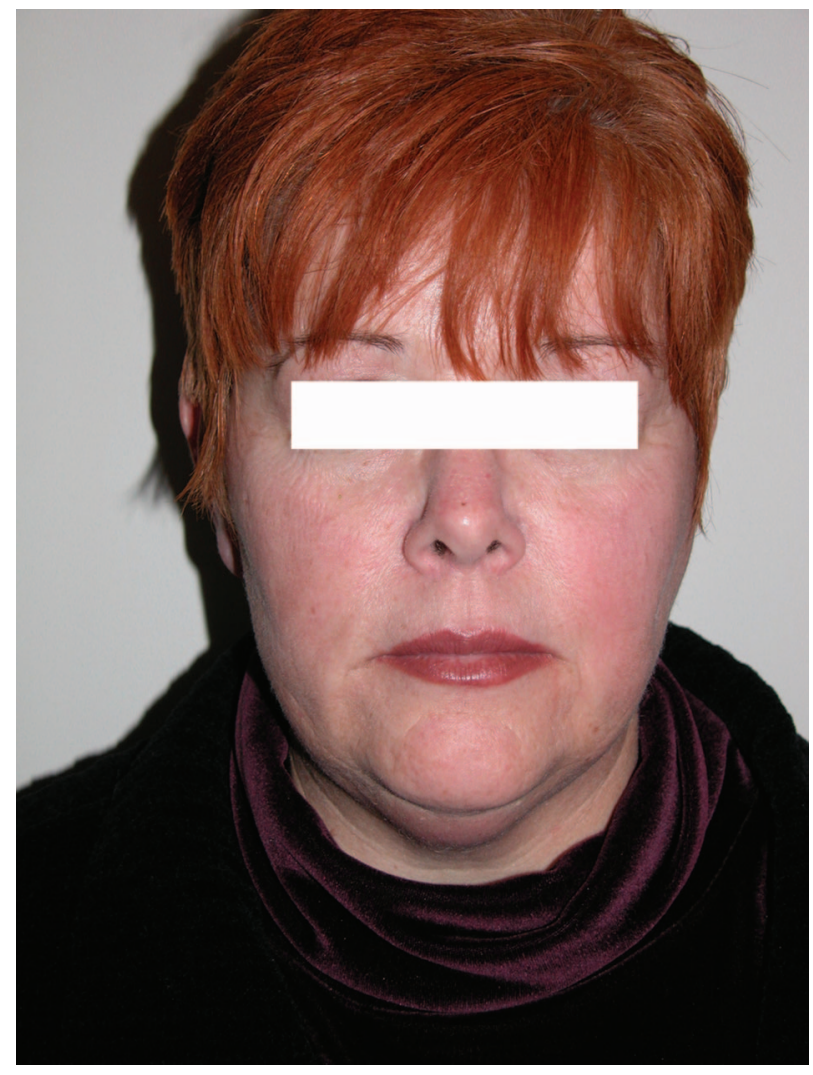

Fig. 3. Frontal view of patient status post right parotidectomy with superficial musculoaponeurotic system flap and fat graft at approximately 9 months after surgery.

had relatively small amounts of tissue removed (mean volume, $7.9 \mathrm{~cm}^{3}$ ) (Table I). Their reported facial asymmetry was $12 \%$ versus $32 \%$ in the control group (Table II). Moreover, both of the study patients complaining of asymmetry had mild defects versus four mild defects and one moderate defect in the control group. Although these results are not statistically significant $(P=.147)$, we suggest that SMAS elevation and resuspension may ameliorate small contour defects. Redraping the SMAS provides little bulk to fill a defect but may resuspend the facial musculature and provide subtle contour improvement. Furthermore, the small sample size in this study may obscure the subtle benefit provided by SMAS elevation.

Several methods including SCM muscle flaps and dermis-fat grafts have been described for parotidectomy reconstruction. ${ }^{1-4}$ The SCM flap is effective but not without risks such as spinal accessory nerve injury. ${ }^{2}$ The use of free fat and free dermis-fat grafts for improvement of facial contour have been described for many situations, but long-term results are controversial. ${ }^{4-6}$ The primary shortcoming of fat grafting has been resorption. For this reason, some authors have advocated the inclusion of dermis in the graft to maintain the subdermal vascular plexus, theoretically optimizing blood flow to the graft and resulting in maximal volume retention. ${ }^{6}$ However, histologic studies have not supported this theory. ${ }^{10}$ Moreover, additional equipment and skill are required to dermabrade the graft. 4,5
Two small series have reviewed the use of dermis-fat grafts for the reconstruction of parotid defects. Nosan et al. ${ }^{4}$ reported on nine cases using a dermis-fat graft used for a parotid defect with a goal of overcorrecting the defect volume by $10 \%$ to $15 \%$ to account for resorption. With a follow-up of 4.5 years, one of nine patients developed a subsequent concave facial defect. Harada et al. ${ }^{5}$ reported on a series of seven patients undergoing a similar procedure with a 24 to 40 month follow-up period. One patient developed a concave deformity in the immediate postoperative period. Conley and Clairmont ${ }^{6}$ reported the use of dermis-fat grafts for reconstruction of posttraumatic and postsurgical defects of the head and neck. It was found that $10 \%$ had near total graft loss, and another $14 \%$ had significant loss in the immediate postoperative period.

Resorption may occur by several different mechanisms. In the immediate postoperative period, grafted adipocytes are subject to ischemic and traumatic necrosis. This can be augmented by complications such as infection and hematoma formation. Longer-term, gradual resorption of fat grafts has also been noted to occur in several reviews. ${ }^{6}$ Finally, volume may be lost secondary not only to resorption adipocytes but their lipid content as well. ${ }^{11}$

In this study, patients reconstructed with fat grafting reported statistically significant improvement in facial symmetry over nonreconstructed patients $(P=.002)$. Furthermore, patients reporting asymmetry reported a lesser degree than controls (Table II). Facial asymmetry was reported in $9 \%$ (3 patients) versus $39 \%$ (15 patients) of the controls. The three patients who noted asymmetry in this group were surveyed at 10 months or longer $(10,30,45$ mo) since surgery. Only two patients in this group were surveyed at less than 10 months postoperatively, and the average length of follow-up was 27.2 (range, 2-74) months, which should be adequate to evaluate for shortterm resorption. Some authors have suggested that resorption may occur gradually over many years, eventually resulting in total resorption. ${ }^{6}$ Although it is difficult to eliminate this as a possibility, our follow-up includes patients up to 74 months, with few reporting asymmetry.

Some degree of resorption during the initial postoperative period is anticipated. For this reason, the defect is overcorrected by $15 \%$ to $20 \%$, which generally provides a reliable outcome. Nevertheless, patients are made aware preoperatively that resorption and resultant facial asymmetry is possible. One patient experienced significant resorption in the immediate postoperative period, resulting in a moderate facial asymmetry. This patient experienced no infection or hematoma; the graft likely underwent liquefaction after implantation.

A number of factors could contribute to the favorable outcomes for fat grafting in this series. Manipulation of the graft and the time between harvest and implantation are minimized to decrease ischemic and mechanical damage to the graft. All grafts were harvested from the infraumbilical abdominal wall, which has proven to be technically easy and yields abundant tissue. In a small number of our patients excluded from this study, submental fat was used. This proved difficult to harvest as a single graft, and results were less favorable. Finally, in each case, the recipient bed was neither previously oper- 
ated nor irradiated. In Conley and Clairmont's ${ }^{6}$ series, where significant resorption was common, the recipient sites were frequently previously operated, irradiated, or scarred from trauma. All of these factors could contribute to poor vascularity of the recipient bed, resulting in poorer graft survival.

Few patients complaining of facial asymmetry in either the study or control groups actually sought treatment for their asymmetry. We would argue that offering reconstruction with SMAS elevation with or without fat graft is an effective and efficient method for minimizing the permanent aesthetic morbidity associated with the procedure, thus improving patient care.

Many of the reconstructive techniques used to improve facial contour also improve Frey's syndrome. ${ }^{1-4}$ Frey's syndrome has a widely varying reported incidence, with clinical symptoms ranging from $10 \%$ to $30 \%$, and positive starch iodine tests may occur in up to $90 \% .^{2,7}$ Some reports have suggested that sub-SMAS flap elevation does not decrease the incidence of Frey's syndrome after parotidectomy. Results are different, however, when the SMAS flap is elevated as a separate layer. Bonnano and Casson ${ }^{8}$ reported no objective Frey's syndrome by Minor's starch iodine testing in 160 patients using this technique. In this series, the incidence of clinically significant Frey's syndrome was decreased using SMAS elevation with or without fat grafting, although this difference was only significant for the fat grafted group (Table II). Also, patients in the study group complained of a lesser severity of symptoms compared with the control group. Although these reconstructive techniques may be effective in preventing or reducing the clinical symptoms of Frey's syndrome; we did not find total clinical avoidance of Frey's syndrome. The primary factor contributing to this may be that the SMAS is sacrificed if the tumor approximates or violates this layer. An attempt is made to repair the SMAS; however, any areas where SMAS is not interposed may develop Frey's syndrome.

A modified rhytidectomy incision was used in study group patients, whereas a standard Blair incision was used in the control groups. Multiple authors using a rhytidectomy incision have demonstrated adequate exposure of the parotid gland and facial nerve without significantly lengthening the time of surgery. ${ }^{9}$ In our series, the incision never limited exposure of either the tumor or facial nerve. The use of a rhytidectomy incision obviates a postoperative neck scar.

Length of follow-up differed significantly in both study groups compared with their respective control groups (Table I). Symptoms of facial asymmetry and Frey's syndrome are unlikely to vary with time in the nonreconstructed control groups. However, length of follow-up is clearly an important factor for fat grafting and could impact results; yet, our data ranges from 2 to 74 months, averaging over 22 months. This provides adequate assessment of short-term fat resorption and suggests that long-term resorption in these patients may be limited as well.

No patient in this series experienced permanent facial weakness (Table II). Transient weakness in all four groups was limited. Two patients in the fat grafted group experienced accumulation of sialoma compared with none in any other group. Auricular numbness was equivalent in the
SMAS elevation group compared with the respective control group, whereas more patients in the SMAS elevation with fat graft had numbness compared with the respective control group. Few patients experienced complications of the fat harvest, which included pain and postoperative scar. No patients experienced local abdominal infections or collections. These results suggest relatively similar risk profiles between the reconstructed and nonreconstructed patients.

Fat graft reconstruction is not offered to patients with suspected or confirmed malignancy given the concern for masking recurrence. SMAS elevation could potentially be used in such cases because this technique is unlikely to obscure palpable recurrence. One patient in this study with preoperative FNA and intraoperative frozen section suggestive of benign disease received a fat graft and was found to have epithelial-myoepithelial carcinoma of final pathology. This rare malignant tumor can be difficult to distinguish from pleomorphic adenoma. ${ }^{12}$ This patient has been monitored with serial clinical examination and MRI and has no evidence of recurrence at 30 months follow-up.

\section{CONCLUSIONS}

SMAS elevation with fat grafting during superficial parotidectomy resulted in a statistically significant decrease in facial asymmetry and symptomatic Frey's syndrome. Although SMAS elevation alone decreased facial asymmetry and symptomatic Frey's syndrome, the difference was not statistically significant. These procedures are technically straightforward and are not associated with increased complications.

\section{BIBLIOGRAPHY}

1. Rappaport I, Allison GR. Superficial musculoaponeurotic system amelioration of parotidectomy defects. Ann Plastic Surg 1985;14:315-323.

2. Casler JD, Conley J. Sternocleidmastiod muscle transfer and superficial musculoaponeurotic system plication in the prevention of Frey's syndrome. Laryngoscope 1991;101: 95-100.

3. Fee WE, Tran LE. Functional outcome after parotidectomy. Laryngoscope 2004;114:223-226.

4. Nosan DK, Ochi JW, Davidson TW. Preservation of facial contour during parotidectomy. Otolaryngol Head Neck Surg 1991;104:293.

5. Harada T, Inoue T, Harashina R, Hatoko M, Ueda K. Dermisfat graft after parotidectomy to prevent Frey's syndrome and the concave deformity. Ann Plast Surg 1993;31: $450-452$.

6. Conley JJ, Clairmont AA. Dermal-fat-fascia grafts. Otolaryngol 1978;86:641-649.

7. Dulguerov P, Quinodoz D, Cosendai G, Piletta P, Marchal F, Lehmann W. Prevention of Frey's syndrome during parotidectomy. Arch Otolaryngol Head Neck Surg 1999;125: 833-839.

8. Bonanno PC, Casson PR. Frey's syndrome: a preventable phenomenon. Plastic Reconstr Surg 1992;89:452-456.

9. Terris D, Tuffo K, Fee W. Modified facelift incision for parotidectomy. J Laryngol Otol 1994;108:574-578.

10. Sawhney Banerjee TN, Chakravarti RN. Behavior of dermal fat transplants. Br J Plast Surg 1969;22:169.

11. Tholpady SS, Aojanepong C, Llull R, et al. The cellular plasticity of human adipocytes. Ann Plast Surg 2005;54: 651-656.

12. Savera AT, Sloman A, Huvos AG, Klimstra DS. Myoepithelial carcinoma of the salivary glands: a clinicopathologic study of 25 patients. Am J Surg Pathol 2000;24:761-774. 